\title{
Studies on Alternating Current Electrolysis
}

\author{
IV. Mathematical Treatment of Reversible Electron Transfer with \\ Alternating Voltage Control and Distorted Current
}

\author{
A. Edward Remick and R. A. Marcus ${ }^{1}$ \\ Departments of Chemistry, Wayne State University, Detroit, Michigan \\ and Polytechnic Institute of Brooklyn, Brooklyn, New York, respectively
}

\begin{abstract}
A mathematical treatment is developed which yields equations relating faradaic current, voltage, and time when an alternating voltage is applied to an electrolytic cell composed of a plane and auxiliary electrodes immersed in a solution containing initially supporting electrolyte and only reversibly oxidizable or reducible species. Both oxidant and reductant are taken to be soluble, and specific adsorption is assumed to be absent. The voltage across that branch of the equivalent circuit through which only faradaic current flows is assumed to be periodic with fixed amplitude and with or without an additional direct applied voltage component; the resultant current is distorted. Diffusion controlled kinetics is postulated, and it is assumed that equilibrium is essentially established at the electrode surface. The equations developed show that a "steady state" (i.e., a periodic state) is quickly attained, yield diagnostic tests of use in establishing the reversible mechanism, make it possible to determine the standard potential, and finally yield for the periodic state a relation between faradaic current and time. These results are then generalized so as to include systems in which the reversible electrochemical step is followed by a sufficiently slow secondary reaction step. One diagnostic result of interest in the latter connection is that the mean faradaic current vanishes in the periodic state, regardless of the amplitude or of the shape of the applied periodic potential, when the follow-up reaction occurs to a negligible extent.
\end{abstract}

The problem of developing a mathematical description of the chemical effects of alternating current on reversible redox systems has been attacked with varying degrees of success by several investigators (1-12) subject to the following restrictions: (a) undistorted voltage and current waves, and/or (b) both components of the redox system must either be present in the solution initially or present at the electrode's surface as the result of superimposed direct current. Berzins and Delahay (8) assumed what in the classification of Delahay, Senda, and Weis (17) may be called alternating voltage (A.V.) control [i.e., controlled sinusoidal potential difference across that branch of the equivalent circuit for one electrode through which only faradaic (f) current is flowing]. They derived an equation for the instantaneous value of the (distorted) faradaic current as a function of time. This equation contained transient terms and a "steady-state" (or, better, a "periodic-state") term. Plane electrodes were involved.

Recently Matsuda and Delahay (13) extended the A.V. control problem to derive an equation for the transient current produced by faradaic rectification after the periodic component had reached a steady state. Their equation was restricted to small values of the A.V. and to solutions initially containing both oxidant and reductant.

1 Alfred P. Sloan Fellow; N.S.F. Senior Post Doctoral Fellow at Institute of Mathematical Sciences, New York University (1960-61).
Koutecky (14), assuming A.V. control, derived an equation for the instantaneous value of the faradaic current as a function of time for the general case of a periodic voltage of optional wave shape and applied it to the special cases of sine waves, square waves, and triangular waves. His equations were largely, although not entirely, concerned with the conditions characteristic of polarography (i.e., dropping electrode), but his work and an initial portion of the present work are related. We shall be concerned with stationary electrodes, with a treatment for (in some ways) slightly more general systems, with a detailed treatment of the statement of the periodic state, and with devising a method of determining the standard potential and other properties ( $m$ and $n$ below) of a reversible electrochemical step in an otherwise irreversible process.

In the present paper we shall discuss a system undergoing A.V. electrolysis with or without superimposed direct applied voltage for the case employing voltage control when the initial concentration of either oxidant or reductant is zero. Clearly, large A.V. amplitudes may be necessary to bring concentrations at the electrode surface into the poised region where reasonable accuracy in potential-concentration relationships may be achieved. ${ }^{2}$

This case is of only limited practical interest for

2 A certain experimental difficulty which, it has been suggested [p. 313 of ref. (17)], could complicate interpretation of the more usual (low amplitude) A. V. control systems, is absent here because of the different method used to interpret the results. 
potentiometrically reversible redox reactions but should be important in the study of oxidations or reductions involving "follow-up" mechanisms, e.g.

$$
\begin{aligned}
& \text { Red } \rightleftharpoons \text { ne }+ \text { Ox (fast) } \\
& \text { Ox } \rightleftharpoons \text { products (slow) }
\end{aligned}
$$

especially when the half-life period of the unstable (or highly reactive) intermediate substance is short compared to the polarographic drop time but long compared to the period of the A.V. in a usable frequency range.

\section{General Assumptions}

In the theoretical treatment along the lines indicated, the following restrictions and assumptions will be made.

(i) A plane electrode is used and conditions for the semi-infinite linear diffusion are achieved.

(ii) A. V. control is employed (A. V. alone or with a superimposed direct voltage component).

(iii) The only depolarizer initially present is the reductant. (This is an unessential restriction, as noted later, and is made only to emphasize the case that is of practical interest here.)

(iv) A supporting electrolyte eliminates migration of the depolarizer and also keeps the $\mathrm{pH}$ constant through buffer action. Convection is assumed absent.

(v) "Spontaneous depolarization" (15) occurs only by diffusion, i.e., the depolarizer concentration at the electrode changes only by diffusion or electron transfer to the electrode and not by secondary chemical reaction or by convection.

(vi) Specific adsorption is absent.

(vii) Electrochemical equilibrium exists between the electrode and the concentrations of the electrochemically active species just outside the region effectively occupied by the electrical double layer.

(viii) In view of the transient nature of faradaic rectification, it will be ignored as a possible source of disturbance of over-all concentration after a steady state (i.e., a periodic state) is achieved.

In principle, the above assumptions are all that are needed. In practice, however, it is desirable that the electrolytic resistance is rendered negligible by the high concentration of supporting electrolyte, and that the working electrodes of the electrolytic cell are a measuring electrode and an unpolarizable auxiliary electrode. A negligible resistance ensures an undistorted voltage wave across the faradaic branch, a very desirable restriction, as discussed later. It should also be noted that when a second faradaic process of the above type occurs, it can be treated as a parallel branch in the equivalent circuit.

Assumption (vii) that chemical equilibrium is achieved with alternating voltage does not imply that the charge transfer resistance (Grahame's $\theta$ ) is zero, but it does mean that the $\theta$ is negligible in comparison with the mass transfer resistance (diffusion) for the experimental conditions, i.e., that the frequency used is low enough to enable close approach to equilibrium in a small fraction of a cycle. The validity of this assumption in the case of a poised ferrocyanide-ferricyanide solution was dem- onstrated by Remick and McCormick (16) who showed that the introduction of the Nernst equation into Grahame's equations for the faradaic admittance (6) adequately predicted the experimentally observed relation of depolarizer concentration to polarization resistance and capacitance up to the highest frequency used, viz., $5000 \mathrm{cps}$. Less reactive systems would be expected to behave reversibly only up to lower frequencies.

The assumption that electrochemical equilibrium exists between the electrode and species just outside the double layer implies that the salt concentration is high, so that the width of the double layer is appropriately small. Were this condition not fulfilled, one would have to include in the diffusion equation of the $\mathrm{i}^{\text {th }}$ electrochemically active species the usual migration term $\left(D_{1} e_{\mathrm{i}} / R T\right)(\partial \phi / \partial x) C_{1}, \phi$ being the potential at point $x$, and $D_{i}, e_{i}$, and $C_{i}$ being the diffusion coefficient, charge and concentration (at $x$ ) of species $i_{.}{ }^{3}$

The system as a whole will also be undergoing a periodic buildup and disappearance of the electrical double layer at the electrode. The total current at any time is the sum of this nonfaradaic current and the faradaic current $i$, calculated below. That is, as discussed by Grahame (6), the additional process of charging and discharging the double layer can be regarded as corresponding to a condenser (of capacity depending on instantaneous voltage) in parallel with the faradaic branch of the equivalent circuit. We consider first the behavior of the faradaic branch.

\section{Boundary Value Problem}

The reversible electrode reaction

$$
\mathrm{Red} \rightleftarrows m \mathrm{Ox}+n e
$$

involves the soluble species Red and $O x$ whose charge types are not specified. In Eq. [1] $m$ and $n$ are rational numbers. The molar concentrations of these species, respectively, will be symbolized by $C_{R}$ and $\mathrm{C}_{\mathrm{ox}}$ and the bulk concentration of Red by $\mathrm{C}_{\mathrm{R}}{ }_{\mathrm{R}} \mathrm{C}_{\mathrm{R}}$ and $C_{o x}$ are functions of the time, $t$, and the distance, $x$, from a plane parallel to the electrode surface but just outside the electrical double layer region. To express diffusion control, Fick's second law will be employed as usual, expressed in terms of concentration rather than activities:

$$
\begin{aligned}
\partial C_{o x}(x, t) / \partial t & =D_{\mathrm{ox}}\left[\partial^{2} C_{o x}(x, t) / \partial x^{2}\right] \\
\partial C_{\mathrm{R}}(x, t) / \partial t & =D_{\mathrm{R}}\left[\partial^{2} C_{\mathrm{R}}(x, t) / \partial x^{2}\right]
\end{aligned}
$$

where $D_{\mathrm{R}}$ and $D_{\mathrm{ox}}$ are diffusion coefficients. Equations [2] and [3] will be solved subject to the initial and

3 This assumption concerning the width of the double layer will often be satisfied even when the assumption would be inaccurate for systems for which a kinetic boundary condition is appropriate (i.e., a boundary condition such as $-D \partial C / \partial x=k C_{0 x}-k^{\prime} C_{R}$ at the elec"rode surface) ", "n this kinetic case, Bockris (26) believed that the zeta potential is largely suppressed at concentrations above ca. $1 \mathrm{~N}$. This conclusion, however, was not substantiated by Breiter, Kleinerman, and Delahay (27) whose calculations (based on a theoretical equation which was found to be in accord with experimental data obtained in the polarographic reduction of iodate ins) indicated Since electron transfer proceeds from positions immediately adjacent to the electrode, we see that for double layer effects to be negligible in the kinetic case, the double layer should be narrower than a few Angstroms. In the electrochemical equilibrium case (assumption vii) however, to satisfy our assumption, it merely suffices that diffusion be rapid across the double layer so that concentration ratios inside it are given by a local Nernst (i.e., Boltzmann) relation. Accordingly, our assumption will often be valid when it is not valid to neglect double 
boundary conditions (and to the assumption of a bounded solution):

$$
\begin{gathered}
C_{\mathrm{R}}(x, 0)=C_{\mathrm{R}}^{o} \\
C_{\mathrm{ox}}(x, 0)=0 \\
D_{\mathrm{ox}} \partial C_{\mathrm{ox}}(x, t) / \partial x+m D_{\mathrm{R}} \partial C_{\mathrm{R}}(x, t) / \partial x=0 \\
\text { at } x=0
\end{gathered}
$$

plus a condition on $C_{0 x}(0, t)$.

If one considers instead of [5] the alternative condition that $C_{0 x}(x, 0)$ equals a nonzero quantity $\mathrm{C}^{\circ}{ }_{\mathrm{ox}}, \mathrm{Eq}$. [7], [13], and [15] remain valid, if $C_{o x}(x, t)$ in [7] is replaced by $C_{o x}(x, t)-C^{\circ}{ }_{o x}$, and $f$ in [13] and [15] by $f-C^{0}{ }^{o x}$. Equation [8] remains intact, but in Eq. [9] and [14] the second $C_{\text {ox }}$ and $f$ should be replaced by $C_{0 x}-C^{o}{ }_{o x}$ and $f-C^{o}{ }_{o x}$, respectively.

In Appendix I, Eq. [2] to [6] are solved by ${ }^{4}$ the Laplace transform method. The solution is given by

$i_{f}=-n F A\left(D_{\mathrm{ox}} / \pi\right)^{1 / 2} \frac{\partial}{\partial t} \int_{o}^{t} C_{\mathrm{ox}}(0, t-\tau) \tau^{-1 / 2} d \tau$

As the boundary condition at $x=0$, we employ the Nernst relation between $C_{o x}(0, t)$ and $C_{n}(0, t)$ for $t>0:^{5}$

$\widetilde{E}_{f} p(\omega t)=E^{\prime}+(R T / n F) \ln \left\{C_{0 \mathrm{x}}(0, t)^{m} / C_{\mathrm{R}}(0, t)\right\}$

where $\tilde{E}_{f}$ is the amplitude of the applied alternating voltage, $p(\omega t)$ is any continuous periodic function of $\omega t$, of period $2 \pi$ and of unit amplitude, and $E^{\prime}{ }_{o}$ is a quantity defined later.

From Eq. [8] and Eq. [24] of Appendix I, it follows that for $t>0, C_{o x}(0, t)$ in Eq. [7] satisfies the relation:

$$
\begin{aligned}
{\left[C_{o x}(0, t)\right]^{m}=\left[C_{\mathrm{R}}^{\circ}-\left(D_{\mathrm{ox}} / D_{\mathrm{R}}\right)^{1 / 2} C_{\mathrm{ox}}(0, t) / m\right] } \\
\\
\exp \left[(n F / R T)\left(\tilde{E}_{f} p(\omega t)-E^{\prime}{ }_{o}\right)\right]
\end{aligned}
$$

The quantity $E^{\prime}$, is defined through the following argument: Since the electrolytic resistance is assumed negligible, the potential difference across the cell is the sum of the two half-cell potentials, $E_{a}+E_{c}$, where $E_{c}$ is the half-cell potential across the auxiliary unpolarizable electrode. If $E_{d}$ is the direct component of the applied potential, we thus have

$$
E_{a}=E_{d}+\tilde{E}_{f} p(\omega t)-E_{c}
$$

Writing $E_{a}$ in terms of its standard potential $E_{o}$ of the reversible step of the half-cell, we have

$E_{a}=E_{o}+(R T / n F) \ln \left\{C_{o x}(0, t)^{m} / C_{R}(0, t)\right\}$

From Eq. [10] and [11], Eq. [8] is obtained with $E^{\prime}$, given by

$$
E^{\prime}{ }_{o}=E_{o}+E_{c}-E_{d}
$$

Delahay, Senda, and Weis (17) have emphasized the desirability that, in specifying the type of con-

\footnotetext{
4 Equation [7] can also be obtained, after a somewhat involved series of substitutions, from the first half of $\mathrm{Eq}$. [21] of ref. (14). However, the derivation given in Appendix I of this paper has eertain advantages for our purposes and is referred to later.

5 The apparent discontinuity between the limit which this $C_{o x}(0, t)$ approaches as $t \rightarrow 0$ and that which Eq. [5] approaches as $x \rightarrow 0$ causes no difficulty. It is indeed a standard type of discontinuity (22) in diffusion and heat conduction problems.
}

trol used, both the alternating and mean components of the current or voltage control should be designated. The argument of the preceding paragraph shows that we are here dealing with the case of A.V. control, with $\bar{E}_{\text {cel1 }}=E_{d}$ (when the mean value of $p(\omega t)$ is zero), i.e., with $\overline{E_{a}}=\overline{E_{c}}-E_{d \cdot}$

It sometimes happens that $\widetilde{E}_{f} p(\omega t)$ is treated as a piecewise continuous function, ${ }^{6}$ for example, when it is treated as a square wave applied potential. Strictly speaking, it is continuous, but is approximated by this discontinuous function. To adapt Eq. [13] to this case, one uses the fact that the derivative of a step function is a Dirac $\delta$-function (19).

\section{Applications of Equations [7] and [9]}

Silverman and Remick (18) observed oscillographically that a periodic state was achieved very rapidly using $\tilde{I}_{\mathrm{ee} 11}$-control, [i.e., A.C. control (17)], plane electrodes, and solutions initially containing only one component of a reversible redox system. We know of no comparable experimental demonstration involving A.V. control; however, Eq. [7] and [9] can be shown to predict the attainment of a periodic state. Moreover, this periodic state is a convenient one for application of the equations to experimental data. Accordingly, we first show how these equations lead to the periodic state.

For this purpose, it is very convenient to introduce a function $f(\omega \theta)$ which agrees with $C(0, \theta)$ for $\theta>0$, but which is the periodic continuation of $C(0, \theta)$ for $\theta \leq 0$.

In Appendix II it is then shown that Eq. [7] can be rearranged to give

$$
\begin{aligned}
i_{t}=n F A\left(\omega D_{\circ \mathrm{x}} / \pi\right)^{1 / 2} & \left\{\int_{0}^{\infty} \frac{\partial}{\partial y}[f(\omega t-y)] y^{-1 / 2} d y\right. \\
& \left.-1 / 2 \int_{\omega t}^{\infty} f(\omega t-y) y^{-3 / 2} d y\right\}
\end{aligned}
$$

where $f(\omega t-y)$ satisfies Eq. [14] for all $t$. Equation [14] is obtained from Eq. [9] by replacing $C_{0 x}(0, t)$ by $f(\omega t-y)$.

$[f(\omega t-y)]^{m}=\left[C^{o}{ }_{R}-(f(\omega t-y) / m)\left(D_{0 x} / D_{R}\right)^{1 / 2}\right]$

$$
\exp \left\{(n F / R T)\left[\tilde{E}_{f} p(\omega t-y)-E^{\prime}{ }\right]\right\}
$$

In Appendix II, it is shown that $f(\omega t-y)$ is bounded by some quantity, $M$ say, so the second integral in [13] tends to zero as $\omega t$ tends to infinity

It may be recalled that a piecewise continuous function is one which has in any finite interval at most a finite number of discontinuities.

7 In the case of piecewise continuous $p(\omega t)$, one may utilize Eq. $[22]$ and $[9]$ as follows. Piecewise continuous $p(w t)$ implies piecewise continuity of the function $C_{0 x}(0, t)$ defined by Eq. [9], and
even Laplace transform $\bar{C}_{0 x}(0, s)$ therefore exists and has "nice properties." Consider now the contour integral expression obtained for (23) to Eq. [22], where $C_{\text {ox }}(0, s)$ has just been described. This expression has the desirable convergence behavior outlined in ref. (23) (uniform convergence in $x$ and in $t$ ) and one may proceed to test as discussed in ref. (23) whether it and the corresponding contour integral for $u(0, t)$ boundary conditions. $\partial C_{0 x}(x, t) / \partial x$ and thereby $i_{f}$ may be obtained then by differentiating under the integral sign. Indeed, some of the proofs in Appendices II and III might well have been shortened through use of the contour integral.

Equations [13] and [15] do not apply at those times $t$ for which $f(\omega t)$ is discontinuous since Eq. [24] of Appendix I (and hence Eq. [91) does not. 
since its majorant, $M \int_{t}^{\infty} y^{-3 / 2} d y$, behaves in this way. Hence, this integral is a transient term. The first integral in [13] is periodic in $\omega t$, because $f(\omega t-y)$ is periodic in wt (as shown in Appendix III), its derivative, therefore, being periodic also, and because its limits are not functions of $t$. This integral, therefore, describes the periodic state.

An equivalent form of Eq. [13], derived in Appendix II, is given by Eq. [15], the first integral being the periodic term and the second being the same transient term as in Eq. [13].

$$
\begin{aligned}
i_{f}=-1 / 2 n F A\left(\omega D_{0 \mathrm{x}} / \pi\right)^{1 / 2}\left\{\int_{o}^{\infty}[f(\omega t)-f(\omega t-y)] y^{-3 / 2} d y\right. \\
\left.+\int_{\omega t}^{\infty} f(\omega t-y) y^{-8 / 2} d y\right\} \quad[15]
\end{aligned}
$$

Using these equations, it is shown, incidentally, in Appendix III that the mean value of the periodic state integral in Eq. [13] or [15] is zero, for arbitrary periodic functions $p(\omega t)$ and for arbitrarily large amplitudes. This theorem generalizes earlier discussions by other investigators for small amplitudes. It should have diagnostic value since it would obviously be false if the irreversible follow-up reaction occurred appreciably.

To examine a possible way for determining $E^{\prime}$ S and other properties, it is convenient to convert the integral for the periodic state into a "reduced" form. That is, we proceed to deduce an "equation of corresponding states" for the faradaic current and for its dependence on applied potential.

We see from Eq. [14] that at any given temperature $f(\omega t-y)$ depends on $n E^{\prime}{ }_{o}, m, C^{{ }^{\prime}}{ }_{R}$ and on the value of the function $n E_{f}$ at the time $\omega t-y$ (taking $\sqrt{D_{\mathrm{ox}} / D_{\mathrm{R}}} \cong 1$ ). Therefore, we may write (for given $T), f(\omega t-y)$ as a function of $C^{\circ}{ }_{R}$, of $n E^{\prime}$, of $m$, of the value of $n E_{f}$ at time $\omega t$, and of $y{ }^{8}$ Similar remarks apply to the $y$-derivative of $f(\omega t-y)$. Accordingly, the periodic state term in either [13] or [15] satisfies the formal relation:

$i_{f}=n F A\left(\omega D_{o x} / \pi\right)^{1 / 2} \int_{0}^{\infty} h\left(y, n E^{\prime}, m n E_{f}, C^{o}{ }^{o}\right) d y$

where the function $h$ can be written in the two equivalent forms (forms which differ only by a quantity whose integral from 0 to $\infty$ vanishes):

$$
\left.\begin{array}{l}
h \equiv y^{-1 / 2} \partial f(\omega t-y) / \partial y \\
h \equiv \frac{1}{2} y^{-8 / 2}[f(\omega t-y)-f(\omega t)]
\end{array}\right\}
$$

Equation [16] is a convenient one for our purposes, for we see from Eq. [13] that at a given temperature, $C^{\circ}{ }_{n}$ and electrode area $A$, a plot of $n E_{f} v s$. $i_{f} / n\left(\omega D_{\mathrm{ox}}\right)^{1 / 2}$ depends only on $n E^{\prime}{ }_{\circ}$ and $m$. Therefore, by adjusting the direct voltage $E_{a}$ and (in multiples of some preassigned amplitude) $\tilde{E}_{f}$, the $E_{f} v s$. $i_{f}$ plot could be made to conform to a standard shape, a shape which, for any preassigned $n E^{\prime}{ }_{0}, n \tilde{E}_{i}, C^{o}{ }_{R}$ and $T$, depends only on $m$. From the value of $E_{d}$ needed to attain this specified $n E^{\prime}{ }_{o}, E_{o}$ could be calculated from Eq. [12], $n$ could be determined from the $\tilde{E}_{f}$

${ }^{8}$ The value of $E_{f}$ at $(\omega t-y)$ is determined if one knows for all $t$ its value at $\omega t$ and if one knows $y$, i.e., $E_{1}(\omega t-y)$ is a function of the function $E_{f}(\omega t)$ and of $y$. needed to attain the standard shape, and $m$ could be determined from the $\mathrm{C}^{\circ}{ }_{\mathrm{B}}$-dependence of the data. (For example, for $m=1$ Eq. [14] shows that $f(\omega t-y)$ is directly proportional to $C^{0}$, so $h$ and hence $i_{f}$ in [16] are directly proportional to $C^{\circ}{ }_{k}$, for any given $E_{f}$.) Again, Eq. [16] provides other diagnostic tests. A plot of $i_{f} / \omega^{1 / 2} v s$. $E_{f}$ should be independent of $\omega$ and of $\tilde{E}_{f}$.

Instead of plotting $E_{f} v s . i_{f}, i_{\text {, can }}$ be plotted $v s$. $\omega t$, after first writing Eq. [16] for the periodic state in the appropriate reduced form. Since $E_{f}=\tilde{E}_{f} p(\omega t)$, the integrand in [16] can be written as a function of $\omega t$ and of $\tilde{n E}$ as well as of $y, n E^{\prime}{ }_{o}, m$, and $C^{\circ}{ }_{R}$. Once again, for any given $C_{R}^{\circ} T$ and electrode area, the adjusting of $E_{a}$ and, in multiples of some amplitude, of $\tilde{E}_{f}$, would lead to a standard shape which depends on $m$. The values of $E_{o}$ and $n$ could then be determined as before, and $m$ could be determined from the $C^{\circ}{ }_{R}$ dependence of the plot. Again, as before, diagnostic tests could be devised: The plot of $i_{f} / \omega^{1 / 2} v s$. $\omega t$ should be independent of $\omega$ and, if $m=1$, should be proportional to $\mathrm{C}_{\mathrm{r}}^{\mathrm{o}}$.

In actual fact, one measures total current rather than only the faradaic branch. The nonfaradaic component is independent of $C^{\circ}{ }_{n}$, so that a plot of $i v s . E_{f}$ or $v s$. $t$ would now be a linear function of $\mathrm{C}^{o}{ }_{\mathrm{R}}$ when $m=1$. On the other hand, no standard shape of a plot of $i v s$. $E$, or $t$ can be attained simply by adjust$\operatorname{ing} E_{a}$ and $\widetilde{E}_{f}{ }^{20}$

Accordingly, in this situation, recognizing that under the assumptions listed earlier, the total current is the sum of the faradaic and nonfaradaic branches and that the nonfaradaic component is independent of $C^{o}$, the value of $i_{f}$ corresponding to any particular phase angle of $E_{f}$ can be obtained by subtracting from the measured, instantaneous current the value of that current when $C^{\circ}{ }_{k}=0$. Examples where this type of subtraction has been made in potentiometric work may be found in ref. (25) and (16). If the condition mentioned earlier that the $i R$ be negligible had not been imposed, no such subtraction process would be permissible for obtaining $i_{f}$, for the $i R$ drop at any given $t$ would cause the instantaneous potential drop across the electrode to depend on $C^{\circ}$, contrary to assumption. Furthermore, since the cell is a nonlinear circuit element, the $i R$ drop through the bulk of the solution would contain harmonics which would invalidate the assumption of a sinusoidal $E_{f}$.

Information about the characteristic behavior of the integral in Eq. [16], and hence about the faradaic current, can be deduced either from numerical integration or from investigation of the properties of known reversible systems. However, some prelimi-

\footnotetext{
${ }^{2}$ To be sure, at small values of $\widetilde{E}_{f}$, points on this plot corresponding to large values of if will not be attained. Note that although if and $E_{f}$ depend on $\widetilde{E}_{f}$, the plot does not.

10 For, if $n E^{\prime}{ }_{\circ}$ and $n \widetilde{E}_{f}$ were each made to conform to their preassigned values by such adjustments, the nonfaradaic current $v s$. $E_{f}$ plot would then differ from system to system: Any two systems normally differ in $E_{o}$ and hence, for a preassigned $n E^{\prime}{ }_{0}$, in $E_{d}$ (cf. Eq. $[12]\}$. But for a given $\mathbb{E}_{a}$, a plot of nonfaradaic current $v s$. $\mathbb{E}_{f}$ would be a standard one only for a preassigned $E_{d}$ and $\widetilde{E}_{f}$.
} 
nary insight into its behavior as far as the dependence of $i_{i}$ on $E_{f}$ in Eq. [16] can be obtained as follows. We shall consider, by way of a concrete example, the important case in which $m=1, D_{\mathrm{ox}}=D_{\mathrm{R}}$, and $p(\omega t)$ is $\sin \omega t$. The integral for the periodic state in Eq. [16] becomes (using it in the form of Eq. [13], after introducing Eq. [14] and performing the differentiation:

$i_{f}=-\frac{n F A C^{\circ}{ }_{n} n F \tilde{E}_{f}}{\left(\pi / \omega D_{o x}\right)^{1 / 2} R T} \int_{0}^{\infty}$

$\underline{\exp \left[n F\left(\tilde{E}_{f} \sin (\omega t-y)-E^{\prime}\right) / R T\right] \cos (\omega t-y) y^{-1 / 2} d y}$

$\left\{1+\left(D_{o x} / D_{R}\right)^{1 / 2} \exp \left[n F\left(\tilde{E}_{f} \sin (\omega t-y)-E^{\prime}{ }_{o}\right) / R T\right]\right\}^{2}$

When $\left|n F E^{\prime}{ }_{o} / R T\right|>>1$ and $\widetilde{E}_{f}<<\left|E^{\prime}{ }_{o}\right|$, the coefficient of $y^{-1 / 2} \cos (\omega t-y)$ in the integrand, is very small at all times, i.e., for all $\left|E_{f}\right| \leq \tilde{E}_{f}{ }^{11}$ Accordingly, $i_{f}$ is then small for all such $E_{f}$, a result expected on physical grounds. In the very special instance that $\widetilde{E}_{r}$ is sufficiently small, the integral in Eq. [18] becomes $^{12}$

$$
\begin{aligned}
\pi^{1 / 2} \cos \left(\omega t-\frac{\pi}{4}\right) \exp \left(-n F E^{\prime}{ }_{o} / R T\right) /[1 \\
\left.+\left(D_{\mathrm{ox}} / D_{\mathrm{R}}\right)^{1 / 2} \exp \left(-n F E^{\prime}{ }_{0} / R T\right)\right]^{2}
\end{aligned}
$$

When $\tilde{E}_{f}$ becomes comparable in magnitude with $E^{\prime}$, however, the current will become large at the appropriate times and, because of the sensitivity of the exponential terms to $\tilde{E}_{f}$, this effect should presumably occur fairly suddenly as $\tilde{E}_{f}$ is increased. Indeed a plot of faradaic current $v s . \tilde{E}_{f}$ may ultimately provide a convenient way for the determination of $E^{\prime}$.

Up to this point, we have restricted our attention to reversible systems. If in addition, the reversible electrochemical step [1] is followed by an irreversible decomposition of the product of [1] to yield an electrochemically inactive substance, Eq. [3] to [6] remain unchanged, as does Eq. [8]. Only Eq. [2] is modified, namely, through the addition of the reaction rate term. If the irreversible step is very slow, this additional term represents only a minor perturbation of Eq. [2]. Physical considerations indicate that the solution for $i_{f}$ should depend continuously on the value of the reaction rate constant in this perturbation term. When this constant is sufficiently small, therefore, the results will approach those previously obtained. That is, our final equations are still applicable, provided the irreversible step is suffi-

\footnotetext{
11 For $E_{o}^{\prime}>0$, the numerator is then very small and the denominator is about unity. For $E^{\prime}{ }_{0}<0$, the denominator is very large compared with unity, so that the first factor in the integrand approximately equals $\left(D_{R} / D_{0 z}\right) \exp \left\{n F\left[E^{\prime}{ }_{o}-\widetilde{E}_{f} \sin (\omega t-y) 1 / R T\right\}\right.$
which is very small.

12 Upon expanding $\cos (\omega t-y)$ and recalling that

$$
\int_{0}^{\infty} y^{-1 / 2} \cos y d y=\int_{0}^{\infty} y^{-1 / 2} \sin y d y=(\pi / 2)^{1 / 2}
$$

and noting that $\cos \omega t+\sin \omega t=\sqrt{2} \cos \left(\omega t-\frac{\pi}{4}\right)$, Eq. [19] is obtained.
}

ciently slow. As mentioned earlier, it is this situation where the present equations are likely to be of the most interest.

Manuscript received May 29, 1961; revised manuscript received April 10, 1962.

Any discussion of this paper will appear in a Discussion Section to be published in the June 1963 JourNaL.

\section{REFERENCES}

1. J. E. B. Randles, Discussions Faraday Soc., 1, 11 (1947).

2. B. Breyer and F. Gutman, ibid., 1, 19 (1947); B. Breyer, F. Gutman, and S. Hacobian, Australian $J$. Sci. Research, A3, 558, 567 (1950); A4, 595 (1951); B. Breyer and S. Hacobian, ibid., A4, 604, 610 (1951); Australian J. Chem., 7, 225 (1954).

3. B. Ershler, Zhur. Fiz. Khim., 22, 683 (1948); K. Rozental and B. Ershler, ibid., 22, 1344 (1948).

4. H. Gerisher, Z. physik. Chem., 198, 286 (1951); ibid., (N.F.) 1, 278 (1954).

5. M. Fournier, Compt. rend., 232, 1673 (1951).

6. D. C. Grahame, This Journal, 99, 370C (1952).

7. P. Delahay and T. J. Adams, J. Am. Chem. Soc., 74,5740 (1952).

8. T. Berzins and P. Delahay, Z. Elektrochem., 59, 792 (1955).

9. I. Tachi and T. Kambara, Bull. Chem. Soc. Japan, 28, 25 (1955).

10. M. Senda and I. Tachi, ibid., 28, 632 (1955).

11. T. Kambara, Z. physik. Chem. (N.F.), 5, 52 (1955).

12. H. Matsuda, Z. Elektrochem., 62, 977 (1958).

13. H. Matsuda and P. Delahay, J. Am. Chem. Soc., 82, 1547 (1960).

14. J. Koutecky, Collect. Czech. Chem. Communs, 21, 443 (1956).

15. M. Wien, Ann. Physik. u. Chem., 58, 37 (1896).

16. A. E. Remick and H. W. McCormick, This Journal, 102, 534 (1955).

17. P. Delahay, M. Senda, and C. H. Weis, J. Am. Chem. Soc., 83, 312 (1961).

18. J. Silverman and A. E. Remick, This Journal, 97, 335 (1950).

19. cf B. Friedman, "Principles and Techniques of Applied Mathematics," p. 440, John Wiley and Sons, Inc., New York (1956).

20. cf A. E. Taylor, "Advanced Calculus," p. 599, Ginn and Co., New York (1955).

21. Ref. (20), p. 529.

22. H. S. Carslaw and J. C. Jaeger, "Conduction of Heat in Solids," 2nd ed., pp. 304-5, 27 or 30, etc., Oxford University Press, London (1959).

23. Ref. (22), pp. $479-480$.

24. Ref. (22), p. 301 .

25. J. E. B. Randles, Discussions Faraday Soc., 1, 11 (1947).

26. J. O'M. Bockris, "Modern Aspects of Electrochemistry," p. 219, Academic Press Inc., New York (1954).

27. M. Breiter, M. Kleinerman, and P. Delahay, J. Am. Chem. Soc., 80, 5111 (1958).

\section{APPENDIX I}

Derivation of Eq. [7]

We let $u(x, t)$ denote the quantity

$$
u(x, t)=C^{0}{ }_{R}-C_{R}(x, t)
$$

Seeking a bounded solution of Eq. [2] to [6], we introduce boundary conditions [4] and [5] into the Laplace transforms of Eq. [2] and [3], respectively, and obtain two differential equations in the domain $x>0$ which can easily be solved to give equations for the transforms, $\bar{u}$ and $\bar{C}_{\mathrm{ox}}$ :

$$
\begin{aligned}
\bar{u}(x, s) & =\bar{u}(0, s) \exp \left(-s^{1 / 2} x / D_{\mathrm{R}}{ }^{1 / 2}\right) \\
\overline{\mathrm{C}}_{\mathrm{ox}}(x, s) & =\bar{C}_{\mathrm{ox}}(0, s) \exp \left(-s^{1 / 2} x / D_{0 \mathrm{x}}{ }^{1 / 2}\right)
\end{aligned}
$$

where $s$ is the Laplace variable. 
Differentiation of these equations with respect to $x$ followed by evaluation at $x=0$ and combination with the transform of Eq. [6] yields

$$
\bar{C}_{\circ \mathrm{x}}(0, s)=\bar{u}(0, s) m\left(D_{\mathrm{R}} / D_{\mathrm{ox}}\right)^{1 / 2}
$$

Taking the inverse transform of Eq. [23] gives

$$
m u(0, t)=C_{o x}(0, t)\left(D_{0 \mathrm{x}} / D_{\mathbf{R}}\right)^{1 / 2}
$$

except at points of discontinuity, using a uniqueness theorem (24).

The instantaneous anodic current, $i_{f}$, is related to the flux according to Eq. [25]

$$
i_{f}=n F A D_{o x}\left[\partial C_{o x}(x, t) / \partial x\right]_{x=0}
$$

wherein we use the convention that an anodic current is negative, and where $A$ denotes the electrode area. The term $\left[\partial C_{0 x}(x, t) / \partial x\right]$, may be calculated from the preceding equations as follows. Differentiating Eq. [22] with respect to $x$ and setting $x=0$, one finds

$$
\begin{aligned}
& {\left[\partial \bar{C}_{o x}(x, s) / \partial x\right]_{x=0}} \\
& =-\bar{C}_{o x}(0, s)\left(s / D_{o x}\right)^{1 / 2}=-s \bar{C}_{o x}(0, s)\left(D_{o x} s\right)^{-1 / 2} \\
& =\left[-s /\left(\pi D_{o x}\right)^{1 / 2}\right] \int_{,}^{\infty} e^{-s t} C_{o x}(0, t) d t \int_{0}^{\infty} e^{-s t} t^{-1 / 2} d t \\
& =\left[-s /\left(\pi D_{o x}\right)^{1 / 2}\right] \int_{0}^{\infty} e^{-s t}\left[\int_{.}^{t} C_{o x}(0, t-\tau) \tau^{-1 / 2} d \tau\right] d t \\
& =-\left(\pi D_{o x}\right)^{-1 / 2} \int_{,}^{\infty} e^{-s t}\left[\frac{\partial}{\partial t} \int_{0}^{t} C_{o x}(0, t-\tau) \tau^{-1 / 2} d \tau\right] d t
\end{aligned}
$$

where we have introduced into (a) the fact that $s^{-1 / 2}$ is simply the Laplace transform of $(\pi t)^{-1 / 2}$. In obtaining (b) we have employed the convolution theorem for Laplace transforms, and in obtaining (c) we have used the standard relation between the Laplace transform of a function (here of $\int_{0}^{t} C_{o x}(0, t-\tau) \tau^{-1 / 2} d \tau$ ) and of its derivative.

It is now immediately apparent from [26c] that

$$
\left[\partial C_{o x}(x, t) / \partial x\right]_{x=0}=-\left(\pi D_{o x}\right)^{-1 / 2} \frac{\partial}{\partial t} \int_{0}^{t} C_{0 x}(0, t-\tau) \tau^{-1 / 2} d \tau
$$

Equation [7] of the text is then obtained from [25] and [27].

\section{APPENDIX II}

Derivation of Eq. [13] and [15] from [7]

We shall consider the behavior of Eq. [7] for times greater than 0 , and we may therefore replace $C_{o x}(0, t-\tau)$ in that equation by the function $f(\omega t-\omega \tau)$, which satisfies Eq. [14] for all values of the argument of $f$, positive or negative. The time derivative in Eq. [7] can then be written as in Eq. [28], after first performing the differentiation and making use of the identity

$$
\begin{gathered}
\partial f(\omega t-\omega \tau) / \partial t=-\partial f(\omega t-\omega \tau) / d \tau \\
\frac{\partial}{\partial t} \int_{1}^{t} f(\omega t-\omega \tau) \tau^{-1 / 2} d \tau \\
=f(0) t^{-1 / 2}-\omega \int_{0}^{t}\left[\frac{\partial}{\partial(\omega \tau)} f(\omega t-\omega \tau)\right] \tau^{-1 / 2} d \tau
\end{gathered}
$$

where $f(0)$ is to be obtained from Eq. [14] by setting wt $-y$ equal to zero there.

Introducing into Eq. [28] a change of variable, $y=\omega \tau$, the right-hand side of the equation becomes:

$$
\begin{aligned}
f(0) t^{-1 / 2}-\omega^{1 / 2} & \int^{\infty}\left[\frac{\partial}{\partial y} f(\omega t-y)\right] y^{-1 / 2} d y \\
& +\omega^{1 / 2} \int_{\omega t}^{\infty}\left[\frac{\partial}{\partial y} f(\omega t-y)\right] y^{-1 / 2} d y
\end{aligned}
$$

It is evident from Eq. [14] that $f(\omega t-y)$ is a bounded function ${ }^{13}$ of $\omega t-y$, so that upon integrating the third term of [29] by parts we get in place of [29]

${ }^{13}$ For any value of $\omega t-y, f(\omega t-y)$ is the positive root of an algebraic equation, all of whose coefficients are finite. The roots of such equations always lie in a finite region of the complex plane.

$$
\begin{aligned}
-\omega^{1 / 3} \int_{0}^{\infty} \frac{\partial}{\partial y} f(\omega t-y) & y^{-1 / 2} d y \\
& +\frac{\omega^{1 / 2}}{2} \int_{\omega t}^{\infty} f(\omega t-y) y^{-3 / 2} d y
\end{aligned}
$$

From Eq. [7], [28], and [30], Eq. [13] then follows.

The first integral in Eq. [13] can also be written in the alternative form

$$
\lim _{\epsilon \rightarrow 0^{+}} \int_{\epsilon}^{\infty} \frac{\partial f}{\partial y}(\omega t-y) y^{-1 / 2} d y
$$

Upon integrating by parts, this term becomes

$$
\begin{aligned}
& -\lim _{\epsilon \rightarrow 0^{+}}\left[f(\omega t-\epsilon) \epsilon^{-1 / 2}-\frac{1}{2} \int_{\varepsilon}^{\infty} f(\omega t-y) y^{-3 / 2} d y\right] \\
& =-\frac{1}{2} \lim _{\epsilon \rightarrow 0^{+}}\left[\int_{\epsilon}^{\infty} f(\omega t-\epsilon) y^{-3 / 2} d y-\int_{\epsilon}^{\infty} f(\omega t-y) y^{-3 / 2} d y\right]
\end{aligned}
$$

where we have introduced the identity

$$
\epsilon^{-1 / 2}=\frac{1}{2} \int_{e}^{\infty} y^{-3 / 2} d y
$$

Rewriting the integrals in [32] as:

$[f(\omega t-\epsilon)-f(\omega t)] \int_{c}^{\infty} y^{-3 / 2} d y$

$$
+\int_{\varepsilon}^{\infty}[f(\omega t)-f(\omega t-y)] y^{-3 / 2} d y
$$

and proceeding to the limit $\epsilon=0$, the first expression in [33] varies as (constant) $(\epsilon)\left(\epsilon^{-1 / 2}\right)$ using the conditions on $f$ specified in footnote ${ }^{14}$ and so vanishes. Introducing the second integral of [33] into [31], Eq. [15] is obtained.

\section{APPENDIX III}

Proof that the Mean Value of $i$ Equals Zero in the Periodic State

In this section we consider the behavior at positive times, and it follows therefore that $t>t_{0}$. Thus, we may replace $C_{o x}$ by $f$.

It is first noted that physical arguments show that there can be only one positive solution of Eq. [14] for $f(\omega t-y)$, even when this equation has more than one solution (i.e., when $m \neq 1$ ). All other solutions must either be negative or partly imaginary. We further note that since $p(\omega t)=p(\omega t+2 \pi), \quad f(\omega t-y)$ and $f(\omega t-y+2 \pi)$ satisfy the same Eq. [14]. Because of the uniqueness of the physically real $f(\omega t-y)$ just mentioned, it then follows that for our positive f's, $f(\omega t-y+2 \pi)=f(\omega t-y)$, i.e., $f(\omega t-y)$ is also a periodic function of $\omega t$ with the same period as $p(\omega t)$.

We next observe that the mean value of the integral describing the periodic state is obtained by averaging it with respect to $\omega t$ over a period of $2 \pi$. Using Eq. [15], the integral which occurs in this average can be written in the equivalent form

$-\frac{1}{4 \pi} \int_{\theta}^{\theta+2 \pi}\left[\lim _{\epsilon \rightarrow 0^{+}} \int_{y=\epsilon}^{\infty}[f(\omega t)-f(\omega t-y)] y^{-3 / 2} d y\right] d(\omega t)$

where $\theta$ is any large value of $w t$. It can easily be shown ${ }^{14}$ that the convergence of the $y$-integral as $\epsilon \rightarrow 0^{+}$is uniform in $\omega t$ and, hence, $\lim$ and $\int$ can be interchanged, using a standard theorem [20]. Thus we obtain

$$
-\frac{1}{4 \pi} \lim _{\epsilon \rightarrow 0^{+}} \int_{\omega t=\theta}^{\theta+2 \pi}\left[\int_{y=\epsilon}^{\infty}[f(\omega t)-f(\omega t-y)] y^{-3 / 2} d y\right] d(\omega t)
$$

14 For $\epsilon$ small and $y$ lying in the interval $(0, \epsilon),|f(\omega t)-f(\omega t-y)|$ $\cong\left|\frac{\partial}{\partial \omega t} f(\omega t)\right| \cdot y$, whence $\left|\int_{0}^{\epsilon}[f(\omega t)-f(\omega t-y)] y^{-3 / 2} d y\right| \leqslant$ $N \int_{0}^{\epsilon} y^{\frac{\partial \omega t}{t}} d y=2 N \epsilon^{1 / 2}$, where $N$, an upper bound to the derivative, is independent of $\omega t$. Actually the differentiability of $f(\omega t)$ needn't have been used. The milder Hölder condition, $f(\omega t)-f(\omega t-y)$
$\leq$ constant $y^{\alpha}$ but where $\alpha>1 / 2$, would have sufficed. 
Because of the readily proven convergence of the $y$-integral with respect to the upper limit $(\infty)$, uniform in $\omega t,[35]$ may be written as

$$
\begin{aligned}
-\frac{1}{4 \pi} \lim _{\varepsilon \rightarrow 0^{+}} \lim _{L \rightarrow \infty} \int_{\omega t=\theta}^{\theta+2 \pi} & {\left[\int_{y=\epsilon}^{L}[f(\omega t)\right.} \\
& \left.-f(\omega t-y)] y^{-8 / 2} d y\right] d(\omega t)
\end{aligned}
$$

The integrals $\int_{c}^{L}[f(\omega t)-f(\omega t-y)] y^{-3 / 2} d y$ and $y^{-3 / 2}$ $\int_{\theta}^{\theta+2 \pi}[f(\omega t)-f(\omega t-y)] d(\omega t)$ exist for any $\epsilon>0$ and

$L$, and the double integral also exists not only for continuous $f(\omega t)$ but also for piecewise continuous $f(\omega t)$. It then follows from a standard theorem [21] that the order of integration can be interchanged. We obtain

$$
\begin{aligned}
-\frac{1}{4 \pi} \lim _{\epsilon \rightarrow 0^{+}} \lim _{L \rightarrow \infty} \int_{\nu=e}^{L} y^{-3 / 2} & {\left[\int_{\theta}^{\theta+2 \pi}[f(\omega t)\right.} \\
& -f(\omega t-y)] d(\omega t)] d y
\end{aligned}
$$

But, since $f(\omega t)$ was seen earlier to be a periodic function of $\omega t$, it is true that

$$
\int_{\theta}^{\theta+2 \pi} f(\omega t) d(\omega t)=\int_{\theta}^{\theta+2 \pi} f(\omega t-y) d(\omega t)
$$

since it is easily shown that the value of a periodic function averaged over its period is independent of the initial value of the phase angle.

We see, therefore, that the ot integral in Eq. [37] must vanish which proves the desired result.

This result of Appendix III can be proven under milder restrictions, such as piecewise continuity of $f(\omega t)$. As pointed out in the text, Eq. [15] for $i_{f}$ is valid for piecewise continuous $f(\omega t)$, except at the isolated points of discontinuity of the latter. The derivation of Eq. [35] from [34] proceeds as before, when points $\omega t$ of discontinuity of $f$ are excluded. The derivation of [37] from [35] and the use of [38] remain valid. Thus, if one defines $i$, arbitrarily but finite at the isolated points of discontinuity of $f(\omega t)$, the mean value of $i_{f}$ is zero in the periodic state for piecewise continuous functions $f(\omega t)$.

\section{SYMBOLS}

$A$, area of electrode's surface, $\mathrm{cm}^{2}$

$C_{o x}$, concentration of oxidant, mole $\mathrm{l}^{-1}$.

$C_{R}$, concentration of reductant, mole ${ }^{-1}$.

$C_{k}^{\prime \prime}$, concentration of reductant in bulk of solution, mole $1^{-1}$.

$D_{o x}$ diffusion coefficient of oxidant, $\mathrm{cm}^{2} \mathrm{sec}^{-1}$

$D_{\mathrm{R}}$, diffusion coefficient of reductant, $\mathrm{cm}^{2} \mathrm{sec}^{-1}$.

$E_{a}$, half-cell potential difference of measuring electrode, $v$.

$E_{c}$, half-cell potential difference of auxiliary, nonpolarizable electrode, v.

$E_{f}$, half-cell potential difference between electrode and the solution just outside of electrical double layer, in Ox-Red system under investigation, v.

$E_{d}$, direct component of applied cell potential, v.

$\widetilde{E}_{f}$, amplitude of the periodic $E_{f}$ wave, v. $E_{f}=E_{f} p(\omega t)$. $E_{0}$, standard potential of the Ox-Red system, v.

$E^{\prime}, E_{o}+E_{0}-E_{d}$.

$\bar{E}_{\text {cel1 }}$, mean cell potential, v.

$f$, function of, (a particular function).

$f$, (as a subscript) faradaic.

$F$, faraday ( 96514 abs.-coulombs g-equiv. ${ }^{-1}$ ).

$h$, function of, (a particular function).

$i$, instantaneous faradaic current, amp.

$m$, number of oxidant molecules produced from oxidation of 1 mole of reductant.

$n$, number of electrons involved in oxidation of 1 mole of reductant

$R$, gas constant $\left(8.3166 \times 10^{7} \mathrm{erg} \mathrm{deg}^{-1} \mathrm{~mole}^{-1}\right)$.

$t$, time, sec.

$T$, absolute temperature.

$u, C^{o}{ }_{R}-C_{\mathrm{R}}$.

$x$, perpendicular distance from a plane which is parallel to the planar electrode of Ox-Red system, but which is just outside the electrical double layer region.

w, angular frequency of alternating voltage, $\sec ^{-1}$.

\title{
Technical Notes
}

\section{The Discharge Properties of $a-\mathrm{PbO}_{2}$ in Dilute $\mathrm{H}_{2} \mathrm{SO}_{4}$ Electrolyte}

\author{
Harry B. Mark, Jr. \\ Department of Chemistry, University of North Carolina, Chapel Hill, North Carolina
}

The discharge properties and discharge mechanism of $\beta-\mathrm{PbO}_{2}$ in $\mathrm{H}_{2} \mathrm{SO}_{4}$ electrolyte have been studied in considerable detail (1-3). There is disagreement as to the mechanism (1-3), but the experimental characteristics of the discharge, such as reduction overpotentials, shape of the potential-time discharge curves, etc., are well known $(1,3)$. Although some of the electrochemical properties (electrodeposition conditions and self-discharge) of $\alpha-$ $\mathrm{PbO}_{2}$ have been the subject of considerable study (4-8), there is little information in the literature concerning the properties of pure $\alpha-\mathrm{PbO}_{2}$ under an applied discharge current $(3,4,9)$. Almost no work has been reported on the properties of pure $\alpha-\mathrm{PbO}_{2}$ in dilute $\mathrm{H}_{2} \mathrm{SO}_{4}$ electrolyte, although a previous paper (3) showed that the discharge properties of $\beta-\mathrm{PbO}_{2}$ change considerably and in an unexpected way as the acid strength of the electrolyte decreases. The discharge capacities of lead storage batteries, which have positive plates containing a mixture of $\alpha-\mathrm{PbO}_{2}$ and $\beta-\mathrm{PbO}_{2}$, have been studied (10), and there is a brief description of the discharge curve obtained in $4.4 \mathrm{M} \mathrm{H}_{2} \mathrm{SO}_{4}$ for an anodized $\mathrm{Pb}$ electrode (6). $\mathrm{Mi}$ crophotographs of the cross section of this electrode and $\mathrm{x}$-ray and electron diffraction patterns seemed to indicate that there is a layer of a mixture of the two modifications of $\mathrm{PbO}_{2}$ between the lead metal and the $\beta-\mathrm{PbO}_{2}$ film which was in contact with the electrolyte (4). The discharge of an electrode, which consists of a layer of $\alpha-\mathrm{PbO}_{2}$ between a layer of $\beta-\mathrm{PbO}_{2}$ and a $\mathrm{Pt}$ support, in a $4.4 \mathrm{M} \mathrm{H}_{2} \mathrm{SO}_{4}$ electrolyte has been reported $(9)$.

In this investigation the discharge in dilute (0.1M) $\mathrm{H}_{2} \mathrm{SO}_{4}$ of $\alpha-\mathrm{PbO}_{2}$ and $\beta-\mathrm{PbO}_{2}$ have been studied, as 